\title{
High-yield thermalized positronium at room temperature emitted by morphologically tuned nanochanneled silicon targets
}

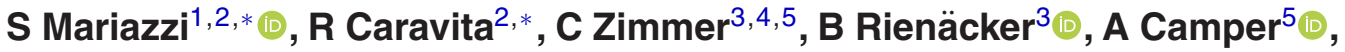 \\ A Belov ${ }^{6}$, G Bonomi $^{7,8}$,, R S Brusa ${ }^{1,2}$, F Castelli $^{9,10}$, G Consolati $^{9,11}{ }^{\text {(D), }}$ \\ M Doser ${ }^{3}$, H Gjersdal ${ }^{5}$, L T Glöggler ${ }^{3}$, F Guatieri ${ }^{1,12}$, S Haider $^{3}$, \\ V Matveev ${ }^{6,13}$, G Nebbia $^{14}$, P Nedelec $^{15}$, D Pagano ${ }^{7,8}$, L Penasa ${ }^{1,2}$, \\ V Petracek ${ }^{16}$, F Prelz $^{9}$, L Povolo ${ }^{1,2}$ (D), O M Rhøne ${ }^{5}$, A Rotondi ${ }^{8,17}$, \\ H Sandaker ${ }^{5}$, N Zurlo ${ }^{8,18}$ and AEgIS Collaboration \\ 1 Department of Physics, University of Trento, via Sommarive 14, 38123 Povo, Trento, Italy \\ 2 TIFPA/INFN Trento, via Sommarive 14, 38123 Povo, Trento, Italy \\ 3 Physics Department, CERN, 1211 Geneva 23, Switzerland \\ 4 Department of Physics, Heidelberg University, Im Neuenheimer Feld 226, 69120 Heidelberg, Germany \\ 5 Department of Physics, University of Oslo, Sem Slandsvei 24, 0371 Oslo, Norway \\ ${ }^{6}$ Institute for Nuclear Research of the Russian Academy of Science, Moscow 117312, Russia \\ 7 Department of Mechanical and Industrial Engineering, University of Brescia, via Branze 38, 25123 \\ Brescia, Italy \\ 8 INFN Pavia, via Bassi 6, 27100 Pavia, Italy \\ 9 INFN Milano, via Celoria 16, 20133 Milano, Italy \\ 10 Department of Physics 'Aldo Pontremoli', University of Milano, via Celoria 16, 20133 Milano, Italy \\ 11 Department of Aerospace Science and Technology, Politecnico di Milano, via La Masa 34, 20156 \\ Milano, Italy \\ 12 FRM II, Technische Universität München, 85747 Garching, Germany \\ 13 Joint Institute for Nuclear Research, Dubna 141980, Russia \\ 14 INFN Padova, via Marzolo 8, 35131 Padova, Italy \\ 15 Institute of Nuclear Physics, CNRS/IN2p3, University of Lyon 1, 69622 Villeurbanne, France \\ 16 Czech Technical University, Prague, Brehov 7, 11519 Prague 1, Czech Republic \\ 17 Department of Physics, University of Pavia, via Bassi 6, 27100 Pavia, Italy \\ 18 Department of Civil, Environmental, Architectural Engineering and Mathematics, University of \\ Brescia, via Branze 43, 25123 Brescia, Italy \\ E-mail: mariazzi@science.unitn.it and ruggero.caravita@cern.ch
}

Received 26 October 2020, revised 15 March 2021

Accepted for publication 9 April 2021

Published 7 May 2021

\section{Abstract}

Nanochanneled silicon targets with high positron/positronium (Ps) conversion rate and efficient Ps cooling were produced. Morphological parameters of the nanochannels, such as their diameter and length, were adjusted to get a large fraction of thermalized Ps at room temperature being emitted into vacuum. Ps cooling measurements were conducted combining single-shot positron annihilation lifetime spectroscopy and Doppler spectroscopy of the $1^{3} \mathrm{~S} \rightarrow 2^{3} \mathrm{P}$ transition. $2 \gamma-3 \gamma$ annihilation ratio measurements were also performed to estimate the positron/Ps conversion efficiency. In a converter with nanochannel diameter of 7-10 nm

* Authors to whom any correspondence should be addressed. 
and depth of $3.89 \mu \mathrm{m}, \sim 28 \%$ of implanted positrons with an energy of $3.3 \mathrm{keV}$ was found to be emitted as Ps with a transverse kinetic energy of $11 \pm 2 \mathrm{meV}$. The reduction of the nanochannels depth to $1.13 \mu \mathrm{m}$, without changing the nanochannel diameter, was found to result in a less efficient cooling, highlighting the presence of Ps reflection from the bottom end of nanochannels.

Keywords: positronium, cooling, silicon, nanostructures

(Some figures may appear in colour only in the online journal)

\section{Introduction}

Positronium (Ps), the bound state of an electron and its antiparticle, the positron $\left(\mathrm{e}^{+}\right)[1]$, is the lightest purely leptonic matter/antimatter atom. Its long-lived ground state, the ${ }^{3} \mathrm{~S}$ state ortho-positronium, o-Ps, has a lifetime of $142 \mathrm{~ns}$ in vacuum and predominantly annihilates into three gamma rays $(3 \gamma)$. The short-lived $1^{1} \mathrm{~S}$ ground state para-positronium, $\mathrm{p}-\mathrm{Ps}$, has a lifetime in vacuum of only 125 ps and its annihilation occurs via $2 \gamma$ emission (as for direct positron-electron annihilation). Production and emission into vacuum of a large amount of Ps with kinetic energies smaller than a few tens of electronvolts is of great interest for several fundamental experiments. These include tests of quantum electrodynamics (QED) [2] and matter/antimatter symmetries [3], probing of gravitational force on Ps atoms excited to metastable levels [4-8] and production of anti-hydrogen by charge exchange reaction [9-11].

In recent years, efficient sources of Ps have been synthesized by exploiting either silica-based disordered porous systems [12-14] or oxidized nanochanneled silicon targets $[15,16]$. Indeed, $\mathrm{Ps}$ is known to be formed in $\mathrm{SiO}_{2}$ irradiated with positrons since 1968 [17]. Subsequent studies have shown that Ps formation in $\mathrm{SiO}_{2}$ occurs both via surface and bulk processes [18-20] with an overall $\mathrm{e}^{+} /$Ps conversion efficiency up to $84 \%$ of the implanted $\mathrm{e}^{+}[21]$. Both processes typically result in Ps atoms emitted into the vacuum with energy of the order of $\approx \mathrm{eV}$ [20]. Emission of colder Ps into the vacuum can be obtained by introducing porous structures in the $\mathrm{SiO}_{2}$ matrix. In these systems, Ps is firstly emitted into the pores. While $1{ }^{1} \mathrm{~S}$ Ps annihilates in a short time, $1{ }^{3} \mathrm{~S}$ Ps, thanks to its relatively long lifetime, can cool (i.e. lose a fraction of its energy) by collisions $[22,23]$ with the inner surfaces of the porous $\mathrm{SiO}_{2}$ material, eventually diffuse along the pore network and be emitted into the vacuum with a significantly lower energy [13, 16, 23-26]. The Ps emission energy into the vacuum depends on the number of collisions with the walls and the energy-loss rate per collision that is determined by the characteristics of the surface of the pores [13, 16, 23-27]. The number of collisions is decided by the depth at which Ps is formed and by the morphology of the pores (namely shape, length, diameter, interconnection and tortuosity) [13, 16, 23-26, 28]. In large pores, the rate of interaction with the inner surfaces is reduced and the cooling time required to reach a given temperature is consequently extended [27, 29]. While in small pores, quantum confinement sets a lowest energy threshold Ps cannot go below $[13,30]$. The result is that the average kinetic energy of Ps emitted by $\mathrm{SiO}_{2}$ porous $\mathrm{e}^{+} / \mathrm{Ps}$ converters is usually higher than the thermal energy of the target $[13,16,23-26]$.

Oxidized nanochanneled silicon $\mathrm{e}^{+} / \mathrm{Ps}$ converters have been introduced in 2010 [15, 16]. These converters offer the possibility to tune the nanochannel diameter and length by acting on the synthesis parameters $[15,31]$. The nanochannel orientation can be changed by choosing the silicon crystal orientation [32] and the depth of Ps formation by the positron implantation energy $[15,16]$. However, even if the physical parameters that contribute to the cooling of Ps are known, it is difficult to match them to optimize the fraction of emitted Ps with an energy at thermal equilibrium with the medium. Targets with nanochannels of 5-8 nm diameter etched in silicon p-type (100) with resistivity of $0.15-0.21 \Omega \mathrm{cm}$ were investigated with $2 \gamma-3 \gamma$ annihilation ratio measurements (in some works also referred as peak-to-valley measurements) and Ps time of flight measurements [16]. Around 35\% of $\mathrm{e}^{+}$ implanted at a mean depth $\langle z\rangle \sim 190 \mathrm{~nm}$ in the converter at room temperature (RT) were found to be emitted as Ps with an average one-directional kinetic energy perpendicular to the surface of $\sim 140 \mathrm{meV}$ [16]. Slower Ps with one-directional kinetic energy of $\sim 96 \mathrm{meV}$ has been observed in the same converters after $\mathrm{e}^{+}$implantation at $\langle z\rangle \sim 470 \mathrm{~nm}\left(7 \mathrm{keV} \mathrm{e}^{+}\right.$ implantation energy). In this case, the emitted Ps amounted to $25 \%$ of the implanted $\mathrm{e}^{+}[16]$. The decrease in the average emission energy has been attributed to the increase in the number of collisions before the emission [16]. Also as, in each collision, the $\mathrm{e}^{+}$of Ps can annihilate with an electron of the walls (pick-off annihilation), the reduction in the fraction of emitted Ps is ascribable to higher number of interactions. Only a small fraction (5\% of implanted positrons) was found to be emitted with thermal energy at RT from these converters.

Converters with larger nanochannels of $10-13 \mathrm{~nm}$ in diameter have been synthetized from silicon p-type (111) with resistivity $0.1-1.5 \Omega \mathrm{cm}$. The choice of larger nanochannels was motivated by the intention to overcome quantum confinement limitations [13, 30]. Silicon with orientation (111) instead of (100) has been chosen because in the former case nanochannels are expected to form a network at $45^{\circ}$ with respect to the surface while in the second case they are expected to be rather perpendicular to the surface [32]. Thus, Ps formed at 
the same depth has to travel a longer path in Si (111) than in $\mathrm{Si}$ (100) converters to reach the surface experiencing a higher number of interactions with the pore walls. Therefore, this choice allows a high emitted Ps fraction to be maintained with low emission energy by lowering the positron implantation energy (i.e. using positrons with a narrower implantation profile [33]). These last converters were used to produce Ps in vacuum for experiments of Ps laser excitation to metastable levels [34-38]. Doppler broadening spectroscopy of the laser-excited $1^{3} \mathrm{~S} \rightarrow 3^{3} \mathrm{P}$ transition [34] and time of flight measurements of Ps in $2^{3} \mathrm{~S}$ state [36] have shown that Ps addressed $20 \mathrm{~ns}$ after $\mathrm{e}^{+}$implantation at $\langle z\rangle \sim 140 \mathrm{~nm}\left(3.3 \mathrm{keV} \mathrm{e} \mathrm{e}^{+}\right.$implantation energy) in the converter at RT has an average one-directional kinetic energy of $\sim 60 \mathrm{meV}$.

In the present work we show how performances of the converters can be drastically improved by etching nanochannels of 7-10 nm of diameter in silicon p-type (111) with resistivity of $0.1-1.5 \Omega \mathrm{cm}$, and implanting $\mathrm{e}^{+}$at a mean depth of $\langle z\rangle \sim 140 \mathrm{~nm}\left(3.3 \mathrm{keV} \mathrm{e}^{+}\right.$implantation energy). The transverse kinetic energy of Ps emitted from these $\mathrm{e}^{+} / \mathrm{Ps}$ converters kept at RT was measured via Doppler broadening spectroscopy of the laser-induced $1^{3} \mathrm{~S} \rightarrow 2^{3} \mathrm{P}$ transition. Single-shot positron annihilation lifetime spectroscopy (SSPALS) [39, 40] was used to quantify the amount of laser-excited Ps. The absolute $\mathrm{e}^{+} / \mathrm{Ps}$ conversion and emission efficiencies into the vacuum were estimated by $2 \gamma-3 \gamma$ annihilation ratio measurements [41]. Our data indicate that a large quantity $\left(\sim 28 \%\right.$ of the implanted $\left.\mathrm{e}^{+}\right)$of Ps emitted from a target with a nanochanneled region $\sim 3.89 \mu$ m deep had a transverse kinetic energy of $11 \pm 2 \mathrm{meV}$, corresponding to the thermal energy in one-dimension. An incomplete thermalization was observed in a converter with a less deep nanochanneled region.

\section{Experimental methods}

\section{1. $e^{+/ P s}$ converters}

Positron/positronium converters were synthetized starting from substrates of silicon p-type (111) with resistivity $0.1-1.5 \Omega \mathrm{cm}$. Nanochannels were produced via electrochemical etching. The etching solution was realized by adding absolute ethanol to a commercial aqueous solution at $48 \%$ of $\mathrm{HF}$ with a volume ratio of 1:3 = HF: ethanol. An etching current of $10 \mathrm{~mA} \mathrm{~cm}^{-2}$ was used. After the electrochemical etching, the samples were cleaned in absolute ethanol $\geqslant 99.8 \%$ and oxidized in air at $100{ }^{\circ} \mathrm{C}$ for $2 \mathrm{~h}$. As shown in reference [15], a fine tuning of the nanochannel diameter can be obtained by different number of etchings in $\mathrm{HF}$ etching solution for $1 \mathrm{~min}$ and re-oxidation in air at $100{ }^{\circ} \mathrm{C}$ for $2 \mathrm{~h}$. In the present work, we produced two targets subject to a single cycle of reetching and re-oxidation (named \#1 in reference [15]). The nanochannel length is determined by the anodization duration [31]. A first target was synthetized with an anodization time of 10 min (hereafter labelled \#1a) and a second one with a time of $4 \mathrm{~min}$ (\#1b in the following).
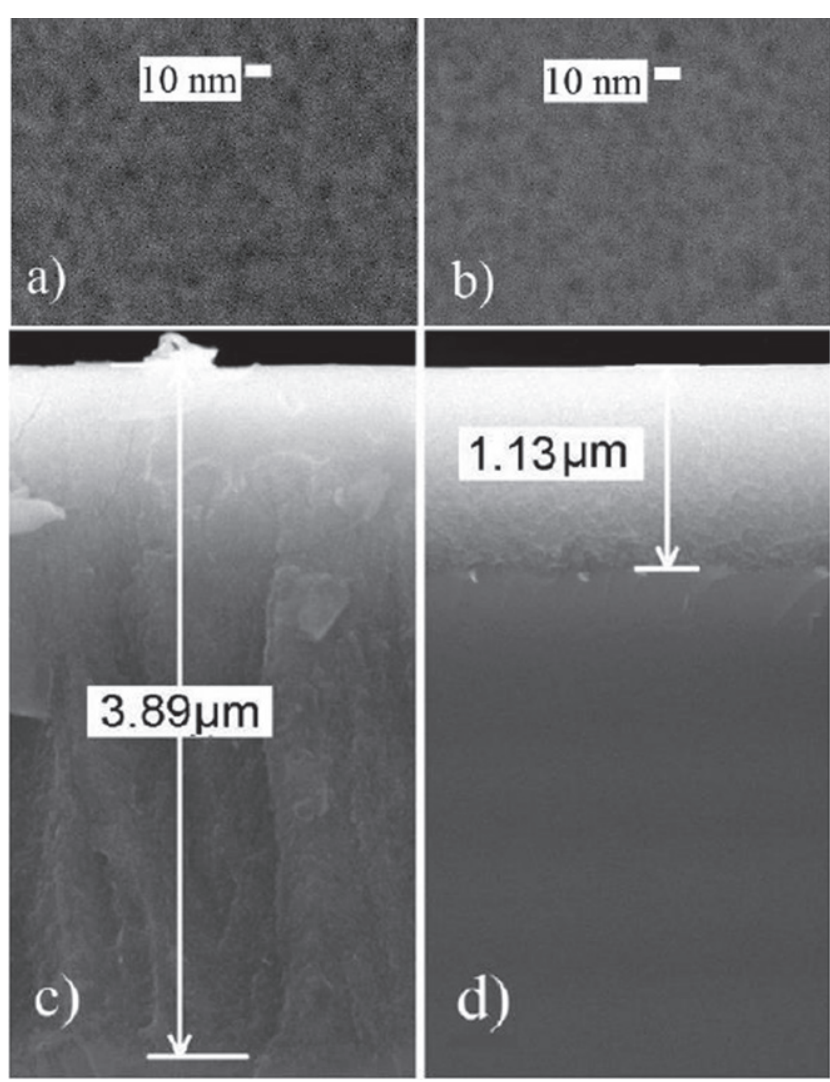

Figure 1. SEM pictures of the surface of samples \#1a and \#1b are reported in (a) and (b), respectively. SEM pictures of the section of samples \#1a and \#1b are shown in (c) and (d), respectively. The thickness of the nanochanneled region is given for both the targets.

The diameter of the nanochannels and the thickness of the nanochanneled region were evaluated by scanning electron microscopy (SEM) carried out by a high resolution JEOL JSM-7001F thermal field emission SEM. Accelerating voltage ranges from 0.5 to $30 \mathrm{kV}$ and its resolution is $1.2 \mathrm{~nm}$ at 30 $\mathrm{kV}$. SEM pictures of the surface of the two targets are reported in figures 1(a) and (b) while the pictures of their sections are reported in figures 1 (c) and (d).

According to the SEM pictures of the surfaces of the converters, the nanochannel diameter ranges between 7 and $10 \mathrm{~nm}$ in both targets. The percentage of the surface not covered by nanochannels amounts to $\sim 80 \%$ of the overall while $\sim 20 \%$ of the surface is etched. This allows us to estimate the density of the nanochanneled region to be around $1.9 \mathrm{~g} \mathrm{~cm}^{-3}$ $\left(=2.33 \mathrm{~g} \mathrm{~cm}^{-3}\right.$ (density of silicon) $\times 0.8$, see also reference [15]) in both the targets. The SEM pictures of the sections of the converters indicate that the thickness of the nanochanneled region is $\sim 3.89 \mu \mathrm{m}$ for sample \#1a and $\sim 1.13 \mu \mathrm{m}$ for \#1b. By comparison with converters synthetized from silicon p-type (100) with resistivity of $0.15-0.21 \Omega \mathrm{cm}$, the nanochanneled region in the present converters is about two times deeper for a comparable anodization time [15]. Several characteristics of the substrate (among them crystal orientation, range of resistivity, feature of the wafer surface, sample size) could be at the origin of the observed difference in the etch rate [42]. 


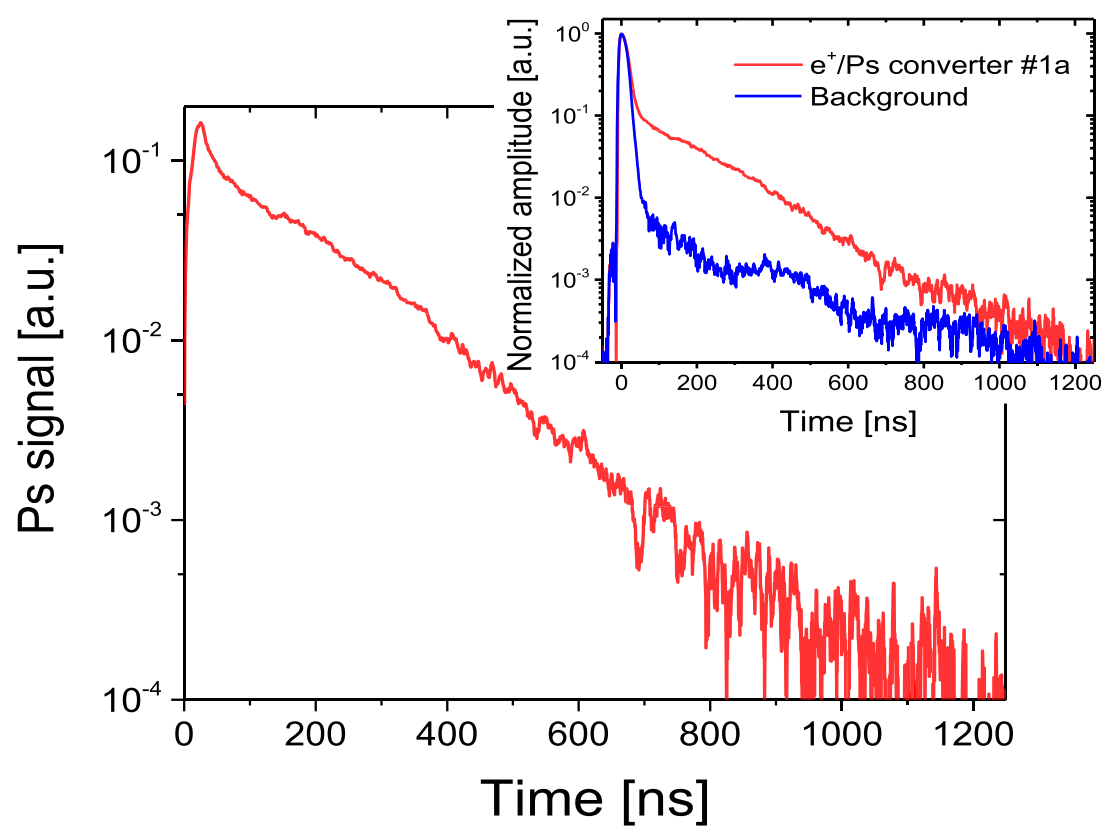

Figure 2. The difference between two SSPALS spectra (Ps signal) measured in a target with Ps formation ( $\mathrm{e}^{+} / \mathrm{Ps}$ converter \#1a) and on the surface of the MCP without Ps formation (background) is reported in the main panel. The two SSPALS spectra, normalized to the peak height, are shown in the inset (converter \#1a in red and background in blue). Positrons were implanted with energy of $3.3 \mathrm{keV}$. Each reported SSPALS spectrum is the average of 40 single shots.

\section{2. $2 \gamma-3 \gamma$ annihilation ratio measurements}

The efficiencies of $\mathrm{e}^{+} / \mathrm{Ps}$ conversion and emission into the vacuum of the two converters were estimated via $2 \gamma-3 \gamma$ annihilation ratio measurements. Measurements were performed with the Trento positron beam [43] by scanning the fraction of implanted $\mathrm{e}^{+}$annihilating into $3 \gamma, F_{3 \gamma}(E)$, as a function of the positron implantation energy, $E$. The term $E$ is related to the mean positron implantation depth, $\langle z\rangle$, through the equation $\langle z\rangle=\frac{40}{\rho} E^{1.6}$, where $\rho$ is the material density [33]. The scan was carried out changing the positron implantation energy from 1 to $21 \mathrm{keV}$. The gamma rays generated by direct $\mathrm{e}^{+}$ annihilations and Ps annihilations were detected by a high purity germanium detector (HPGe) at a distance of about $3.5 \mathrm{~cm}$ from the samples. The HPGe detector had $45 \%$ efficiency and $1.4 \mathrm{keV}$ energy resolution at $511 \mathrm{keV}[41,44]$. The term $F_{3 \gamma}(E)$ was calculated as the ratio of the $2 \gamma$ annihilations in the $511 \mathrm{keV}$ annihilation peak and the $3 \gamma$ annihilations in the region between 410 and $500 \mathrm{keV}$ [41] after calibration with a Ge crystal held at $1000 \mathrm{~K}$ to have $0 \%$ and $100 \%$ Ps formation in the present experimental conditions [41, 45]. The error on $F_{3 \gamma}(E)$, both due to $0 \%$ and $100 \%$ evaluation, was estimated to be less than $3.5 \%$ [15, 45]. Further details about the measurement system and the technique are given elsewhere [15].

In order to guarantee a constant Ps production, the converters were thermally treated in situ, in ultra-high vacuum at $100{ }^{\circ} \mathrm{C}$ for $30^{\prime}$ to remove eventual contaminants before the measurements $[38,46]$.

\subsection{Ps kinetic energy measurements: SSPALS measurements and Ps laser excitation}

The transverse kinetic energy of the Ps emitted by the converters was probed by exploiting the Doppler broadening of the Lyman- $\alpha$ resonance. The measurement was performed as follows. Bursts containing $\sim 10^{7} \mathrm{e}^{+}$were prepared using the AEgIS $\mathrm{e}^{+}$-system at CERN and implanted into the converters with an energy of $3.3 \mathrm{keV}$. Briefly, $\mathrm{e}^{+}$were produced by a ${ }^{22} \mathrm{Na}$ source, slowed by a solid Ne moderator [47] to a kinetic energy of a few eV, trapped and cooled in a Surkostyle trap through the use of buffer gas [48]. Subsequently, $\mathrm{e}^{+}$ were moved to a second trap (accumulator) where the positron plasma containing $\sim 10^{7} \mathrm{e}^{+}$was stored and then extracted by fast pulsing the electric potential on the trap electrodes in the form of $20 \mathrm{~ns}$ bunches. The positron cloud was then magnetically transported in the direction of a chamber dedicated to Ps experiments. Here positrons were further compressed to about $7 \mathrm{~ns}$ in time (FWHM) using a 24-electrode buncher $[49,50]$ and implanted onto the converter with the final kinetic energy of $3.3 \mathrm{keV}$ (see references $[34,35,50]$ for details). The positron spot was of $\sim 3 \mathrm{~mm}$ in diameter [50]. In the present measurements, the target was kept at RT.

The time distribution of $\gamma$ rays emitted by $\mathrm{e}^{+}$and Ps annihilations (SSPALS-spectrum $[39,40]$ ), was acquired with the same procedure used in references [34, 35]. A $20 \times 25 \times$ $25 \mathrm{~mm} \mathrm{PbWO}_{4}$ scintillator, coupled to a Hamamatsu R11265100 photomultiplier tube and digitized by an HD4096 Teledyne LeCroy oscilloscope, was placed $40 \mathrm{~mm}$ above the target. 

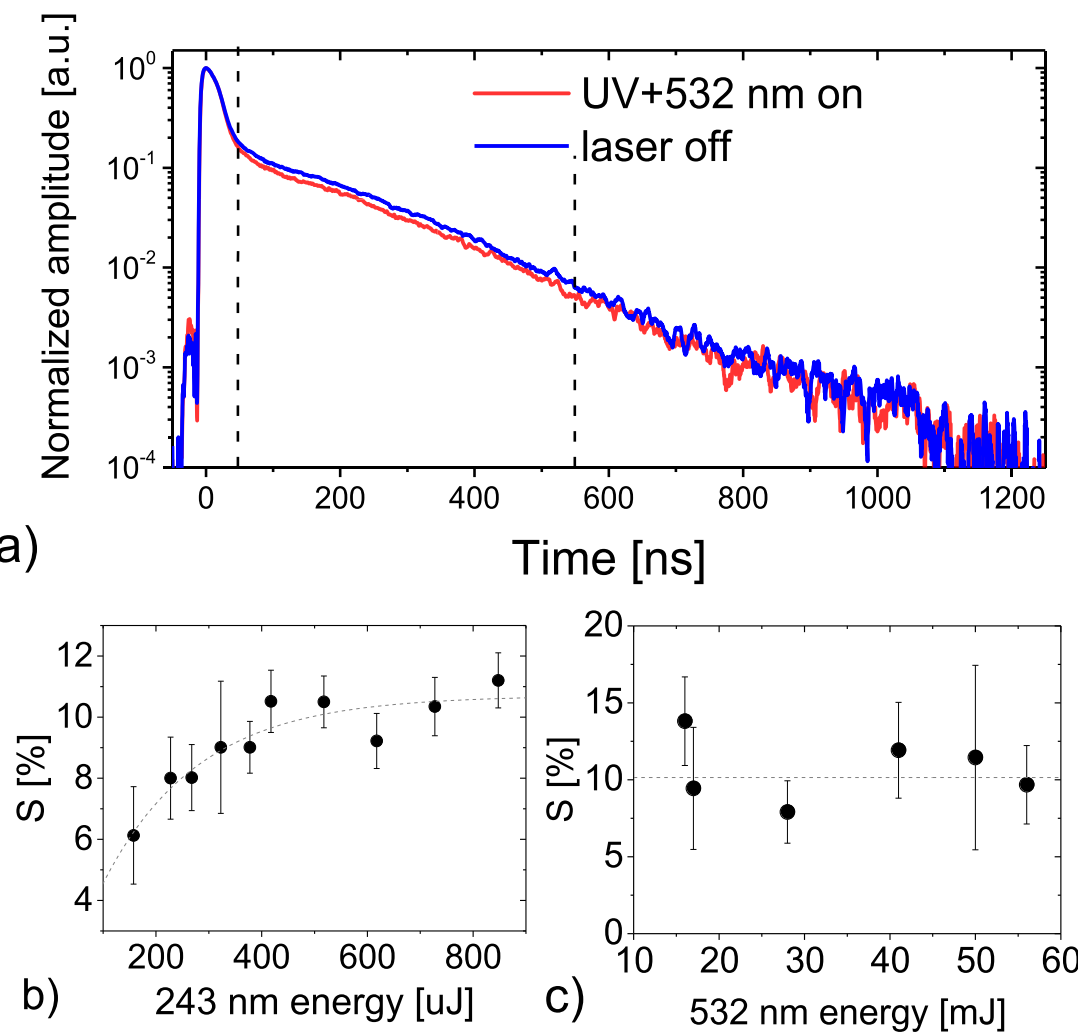

Figure 3. (a) SSPALS spectra of Ps (emitted into vacuum by converter \#1a) with laser off in blue and UV + VIS lasers on $(243.01 \mathrm{~nm}+$ $532 \mathrm{~nm}$ ) in red. The UV energy was $700 \mu \mathrm{J}$ while VIS energy was $50 \mathrm{~mJ}$. Each spectrum is the average of 40 single shots. The vertical dashed lines mark the area between 50 and $550 \mathrm{~ns}$ from the prompt peak used to evaluate the $S$ parameter. $S$ parameter vs UV energy (with VIS energy set at $50 \mathrm{~mJ}$ ) are shown in panel (b) and VIS energy (with UV energy set at $500 \mu \mathrm{J}$ ) in panel (c). The dashed lines are guide for the eye.

An example of two SSPALS spectra, one measured in the absence of Ps formation (by implantation of $\mathrm{e}^{+}$on the surface of a microchannel plate, MCP, placed in the experimental chamber [50]) and one in the presence of Ps formation (by implantation of $\mathrm{e}^{+}$into the converter\#1a), is shown in the inset of figure 2. In the absence of Ps formation, SSPALS spectra present a prompt peak, given by the fast $2 \gamma$ annihilations of $\mathrm{e}^{+}$ implanted in the target. On the right side of the peak, the signal quickly decreases and reaches the noise level in less than $100 \mathrm{~ns}$. The small structures in the spectrum at longer times are photomultiplier ion after pulses $[39,40]$. In the presence of Ps formation, SSPALS spectra show a long tail on the right side of the prompt peak. Such a tail is dominated by the $3 \gamma$ decays of Ps emitted into vacuum. The Ps signal is the difference between the two spectra normalized to the peak height (see main panel in figure 2).

Vacuum-emitted Ps atoms were probed by photo-excitation and photo-ionization, by introducing two laser pulses: an ultraviolet (UV) at $243 \mathrm{~nm}$ and a visible (VIS) at $532 \mathrm{~nm}$. The UV drives the $1^{3} \mathrm{~S} \rightarrow 2^{3} \mathrm{P}$ transition and the VIS immediately photo-ionizes the excited fraction. The broadband $243 \mathrm{~nm}$ pulse was produced by frequency doubling a broadband $486 \mathrm{~nm}$ pulse in a barium borate crystal, produced by a commercial Continuum ND4600 amplified dye laser. The dye oscillator cavity and amplifier were operated with a mixture of
Coumarin 480 and Coumarin 500 dissolved in ethanol in optimal proportions $\left(50 \mathrm{ml}\right.$ of Coumarin $500\left(2 \mathrm{~g} \mathrm{l}^{-1}\right)+150 \mathrm{ml}$ of Coumarin $480\left(1 \mathrm{~g} \mathrm{l}^{-1}\right)+300 \mathrm{ml}$ ethanol for the oscillator, $20 \mathrm{ml}$ Coumarin $500\left(2 \mathrm{~g} \mathrm{l}^{-1}\right)+80 \mathrm{ml}$ Coumarin 480 $\left(1 \mathrm{~g} \mathrm{l}^{-1}\right)+450 \mathrm{ml}$ ethanol for the amplifier), as described in details in reference [51]. The dye system was pumped by a frequency-tripled commercial $1064 \mathrm{~nm}$ CNI Model LPS-L532 Q-switched ND:YAG pump, routinely producing $355 \mathrm{~nm}$ laser pulses of $10 \mathrm{~ns}$ temporal FWHM and $100 \mathrm{~mJ}$ energy, pumping the dye laser. The $243 \mathrm{~nm}$ peak energy was $\sim 900 \mu \mathrm{J}$ with fresh dye solutions and maximum pump power. The UV beam, with an astigmatic Gaussian spatial profile of $1.0 \times$ $2.0 \mathrm{~mm}$ FWHM $(\sim 1.8 \times 3.7 \mathrm{~mm}$ full width at tenth of maximum, FWTM) in the horizontal and vertical directions respectively and with a Gaussian temporal profile of 8 ns FWHM. The bandwidth of the $243 \mathrm{~nm}$ pulses was $50 \mathrm{GHz}$. The intense $532 \mathrm{~nm}$ pulses were produced by frequency doubling the first harmonic of an EKSPLA NL303-HT laser pump in a KDP crystal, producing up to $65 \mathrm{~mJ}$ of $532 \mathrm{~nm}$ in a circular, tophat spatial profile beam of $\sim 12 \mathrm{~mm}$ FWHM with a Gaussian temporal profile of 4 ns FWHM. The use of two independent laser pumps allowed the pulses to be independently triggered and tuned with a $1 \mathrm{~ns}$ resolution. The mutual jittering between the two laser pulses was measured to be $<2 \mathrm{~ns}$ in a typical experimental run. Both laser beams were aligned grazing the surface of the $\mathrm{e}^{+} / \mathrm{Ps}$ converter. 


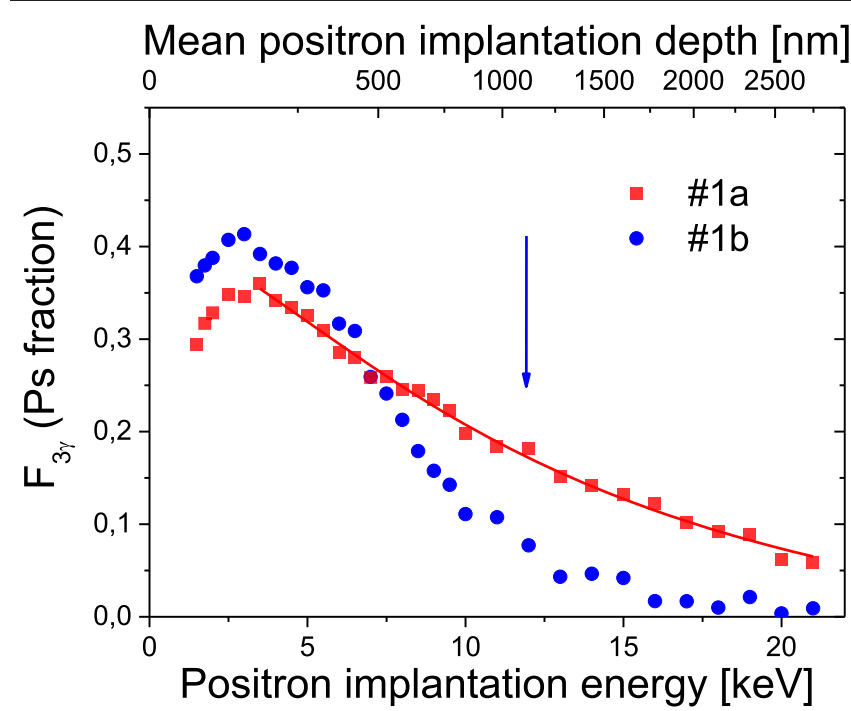

Figure 4. Fraction of implanted $\mathrm{e}^{+}$annihilating via $3 \gamma, F_{3 \gamma}$, vs positron implantation energy $E$ for the converter \#1a (red squares) and \#1b (blue circle). The vertical arrow marks the upper boundary of the nanochannelled region of \#1b. The one of \#1a is out of the scale (see figure 1). The continuous line through the points of \#1a is the best fit obtained by the diffusion model described in reference [15] (see text).

The $1^{3} \mathrm{~S} \rightarrow 2^{3} \mathrm{P}$ laser excitation and photoionization results in a decrease of the Ps population decaying via three gammas. An example is reported in figure 3(a) where SSPALS spectra of Ps emitted into vacuum by target \#1a with laser off and $\mathrm{UV}+$ VIS lasers on are shown. In this measurement, the UV wavelength was set on resonance at $\lambda_{0}=243.01 \mathrm{~nm}$ (in vacuum) and both lasers were shot with a delay of $10 \mathrm{~ns}$ from the positron implantation. Each reported SSPALS spectrum is the average of 40 single shots. The change in the Ps population induced by the interaction with the two laser pulses was quantified by using the $S$ parameter evaluated as:

$$
S=\left(f_{\text {off }}-f_{\text {on }}\right) / f_{\text {off }},
$$

where $f_{\text {off }}$ and $f_{\text {on }}$ are the averages of the normalized areas $f_{\text {off }-i}$ and $f_{\text {on }-i}$ below the $i$ th SSPALS shot, calculated between 50 and $550 \mathrm{~ns}$ from the prompt peak with lasers off and on, respectively [35-37].

The saturation laser energy of both $1^{3} \mathrm{~S} \rightarrow 2^{3} \mathrm{P}$ and $2^{3} \mathrm{P} \rightarrow$ ionization transitions was studied by measuring the $S$ parameter as a function of the UV and VIS energy, respectively (see figures 3(b) and(c)). Also these measurements were performed on sample \#1a. The measurements show that $243 \mathrm{~nm}$ laser is in saturation for energy above $\sim 500 \mu \mathrm{J}$ while the $532 \mathrm{~nm}$ laser saturates above $\sim 15 \mathrm{~mJ}$.

The Doppler broadening of the Lyman- $\alpha$ resonance of Ps was studied by acquiring the $S$ parameter as a function of the UV laser wavelength $[13,52,53]$. The wavelength of the UV pulse $(\lambda)$ was varied around the resonance between 242.7 and $243.2 \mathrm{~nm}$. Doppler broadening experiments were conducted keeping the energy of the UV laser pulse around $700 \mu \mathrm{J}$ and the VIS energy around $50 \mathrm{~mJ}$ to guarantee the saturation of the $1^{3} \mathrm{~S} \rightarrow 2^{3} \mathrm{P}$ and $2^{3} \mathrm{P} \rightarrow$ ionization transition, respectively.

\section{Results and discussion}

\subsection{Estimation of Ps amount emitted into vacuum}

The fraction $F_{3 \gamma}(E)$ of implanted positrons annihilating via $3 \gamma$ vs positron implantation energy $E$ is shown in figure 4 for both the studied converters. The corresponding mean positron implantation depth in the converters, assuming the density $\rho$ $=1.9 \mathrm{~g} \mathrm{~cm}^{-3}$, is shown as the upper abscissa. In both targets, $F_{3 \gamma}(E)$ tops out at $E \sim 3-3.5 \mathrm{keV}$. The maximum value is $\sim 0.35$ and $\sim 0.41$, in \#1a and \#1b, respectively.

The slight decrease of $F_{3 \gamma}(E)$ observed at $E$ lower than $2-3 \mathrm{keV}$ is attributable to both the presence of a lower Ps formation at low positron implantation energies [54] and Ps escaping from the nanochannels after only few collisions with walls and hence with a higher kinetic energy. These Ps atoms can annihilate far-away from the HPGe detector and be detected with a reduced efficiency (see reference [15] for a more detailed explanation).

At positron implantation energy higher than $3-3.5 \mathrm{keV}$, $F_{3 \gamma}(E)$ shows a progressive decrease that is steeper in \#1b than in \#1a. The observed decrease is due to the presence of two contributions: (i) at high $E$, the fraction of Ps able to escape from the nanochannels is reduced by the $2 \gamma$ pick-off annihilations of Ps with an electron of the nanochannel walls and (ii) at high $E$, a fraction of implanted $\mathrm{e}^{+}$annihilates in the unetched Si bulk where Ps formation does not occur. The weight of the two contributions is determined by the Ps diffusion length in the nanochannels and by the thickness of the nanochanneled region.

In order to extract the Ps diffusion length in the nanochannels and the fraction of Ps emitted into the vacuum, the $F_{3 \gamma}(E)$ curve measured in \#1 a was fitted above $E=3.5 \mathrm{keV}$ with the diffusion model described in reference [15]. The fit was performed on the converter with the thicker nanochanneled region because the model assumes nanochannels with infinite length and it does not consider the decrease of Ps formation with the implantation depth due to the effect (ii). The best fit (continuous line in figure 4) points out that the diffusion length in the nanochannels of \#1a is $1200 \pm 200 \mathrm{~nm}$. This diffusion length is around 50\% longer than the one observed in nanochannels of similar diameter but synthetized in Si p-type (100) with resistivity of $0.15-0.21 \Omega \mathrm{cm}$ [15]. The found diffusion length is well shorter than the thickness of the nanochanneled region in $\# 1 \mathrm{a}$, while it is longer than that in the nanochannels of \#1b. This confirms the expectation that in \#1a the effect (i) is predominant, while in \#1b the implantation of $\mathrm{e}^{+}$in the Si bulk is significant and makes the decrease of $F_{3 \gamma}(E)$ steeper than in \#1a. Moreover, the fit indicates that the contribution to $F_{3 \gamma}$ given by Ps annihilating via $3 \gamma$ inside the pores is negligible [15]. Thus, the observed $F_{3 \gamma}$ signal at each $E$ is almost entirely given by Ps back diffused to the surface and emitted into the vacuum. As the nanochannels diameter is found to be identical in the two targets (figure 1) and the interconnectivity of the channel is expected to be the same due to the production procedure, also the $F_{3 \gamma}$ signal measured in $\# 1 \mathrm{~b}$ is expected to be dominated by Ps emitted into vacuum. 


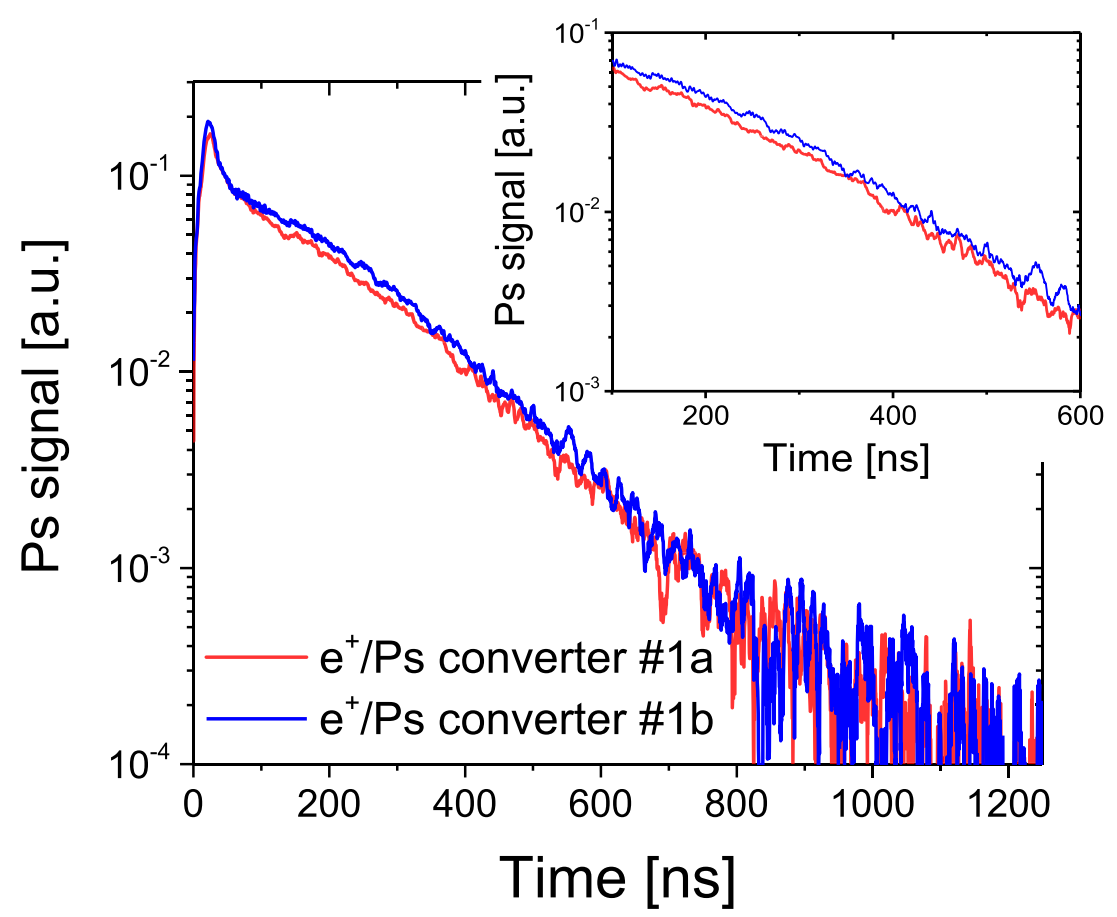

Figure 5. Ps signal estimated as difference of SSPALS for a target with Ps formation and the MCP without Ps formation. Ps signals for $\mathrm{e}^{+} /$Ps converter \#1a (red curve) and \#1b (blue curve) are reported. A detail is shown in the inset.

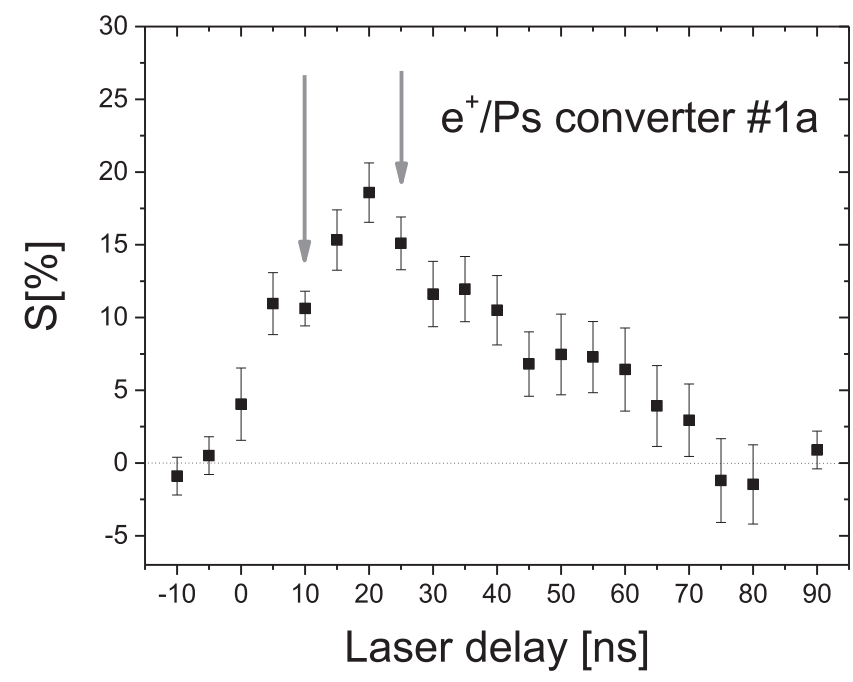

Figure 6. $S$-parameter as a function of the $\mathrm{UV}+532 \mathrm{~nm}$ laser delay with UV laser on resonance. The measurement was performed on converter \#1a. The vertical arrows mark the time delay where the $1^{3} \mathrm{~S} \rightarrow 2^{3} \mathrm{P}$ transition line shapes for Ps emitted from the two converters were measured (see text).

Consequently, the higher $F_{3 \gamma}$ signal observed in \#1b than in \#1a at $E \sim 3-3.5 \mathrm{keV}$ could be ascribed to the different nanochannel length and to the reflection from the nanochannel bottom end [55] that in the thinner \#1b target can result in a more abundant emission into the vacuum.

SSPALS measurements confirm that Ps production given by converter \#1b is slightly larger than the one by \#1a in the proximity of their $F_{3 \gamma}(E)$ maximum (the spectra were acquired at $E=3.3 \mathrm{keV},\langle z\rangle \sim 140 \mathrm{~nm})$. Ps signals in each converter, estimated as difference of SSPALS curves with Ps formation and the background without Ps formation, are shown in figure 5. By comparing the areas between 0 and 1200 ns below the two curves, the Ps signal measured in $\# 1 \mathrm{~b}$ results $\sim 16 \%$ larger than the one observed in \#1a. The observed difference is in agreement with the one indicated by the $F_{3 \gamma}(E)$ measurements.

\subsection{Ps mean velocity evaluation}

In order to find the time at which the largest fraction of Ps is laser excited, a scan of the $S$ parameter as a function of the delay of the two synchronized lasers (UV wavelength set on resonance) with respect to the positron implantation instant was performed. The curve measured for converter \#1a is reported in figure 6 .

The vertical arrows in figure 6 mark the delays at which the transverse kinetic energy of the emitted Ps was measured (10 ns and $25 \mathrm{~ns}$ of delay, respectively). The two delay times have been chosen to sample the velocity of emitted Ps in the proximity of the time at which the maximum Ps laser excitation occurs (around $20 \mathrm{~ns}$ ).

The scan of $S$ parameter as a function of the UV laser wavelength, performed following the procedure reported in section 2.3, is shown in figure 7. Each $S$ value was calculated according to equation (1) from a set of 80 SSPALS single-shot spectra (40 with lasers on and 40 with lasers off acquired in alternated mode). The line shapes of the $1^{3} \mathrm{~S} \rightarrow 2^{3} \mathrm{P}$ transition, measured in \#1 a at $10 \mathrm{~ns}$ and $25 \mathrm{~ns}$, are reported in figures 7(a) and (b), respectively. The measurements performed in \#1b at $10 \mathrm{~ns}$ and $25 \mathrm{~ns}$ are shown in figures 7(c) and (d), respectively. As the width of the $1^{3} \mathrm{~S} \rightarrow 2^{3} \mathrm{P}$ transition is dominated by Doppler broadening, it can be used to obtain the Ps velocity in the direction of the UV laser. The experimental data are 

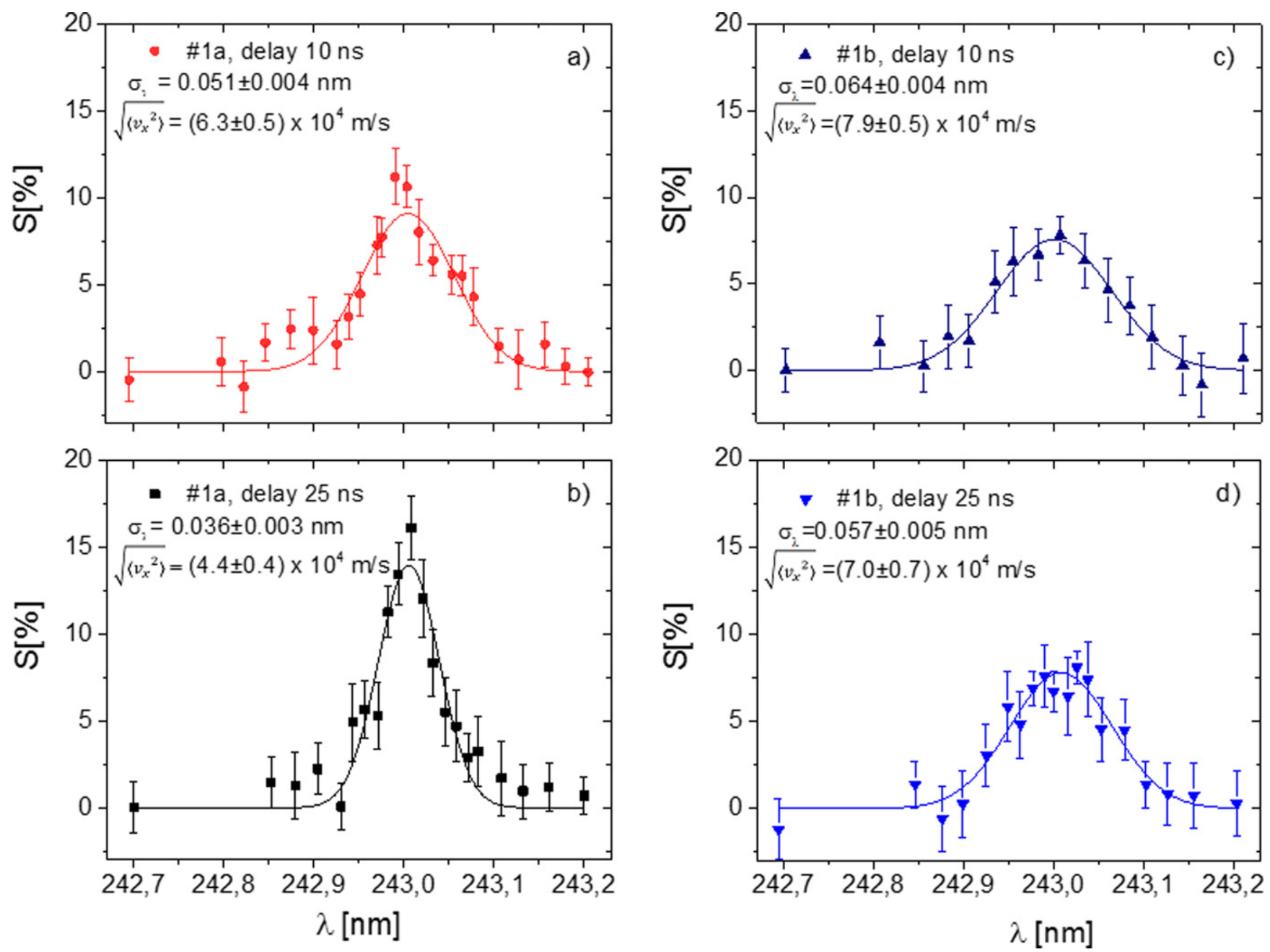

Figure 7. $1^{3} \mathrm{~S} \rightarrow 2^{3} \mathrm{P}$ transition line shapes for Ps emitted from different converters and with different delays between the $\mathrm{e}^{+}$implantation and the laser shot. The transition lines measured in \#1a at $10 \mathrm{~ns}$ and $25 \mathrm{~ns}$ of laser delay are shown in (a) and (b), respectively. Transition lines measured in the converter \#1b at $10 \mathrm{~ns}$ and $25 \mathrm{~ns}$ of laser delay in (c) and (d), respectively. The measurements were performed by implanting positrons with an energy of $3.3 \mathrm{keV}(\langle z\rangle \sim 140 \mathrm{~nm})$. The solid lines are Gaussian fits. The sigma corresponding to each transition line and the relative RMS Ps velocity is reported (see text).

Table 1. $S$ value at resonance and average velocity of Ps measured at 10 and $25 \mathrm{~ns}$ of lasers delay in converter \#1a and \#1b. The angle of the spherical wedge, $\theta$, excitable with the UV laser bandwidth $(\sim 50 \mathrm{GHz})$ and the corresponding excitation efficiency, $d$, are also reported (see text).

\begin{tabular}{lccccc}
\hline Converter & Delay time $(\mathrm{ns})$ & $S(\%)$ at resonance & Average velocity $\left(10^{4} \mathrm{~m} \mathrm{~s}^{-1}\right)$ & Wedge angle $(\theta)$ & Excitation efficiency $(d)$ \\
\hline \multirow{4}{*}{$\# 1 \mathrm{a}$} & 10 & $10.4 \pm 0.8$ & $\sim 6.3$ & $\pm 12^{\circ}$ & $\sim 0.133$ \\
& 25 & $13.9 \pm 1.0$ & $\sim 4.4$ & $\pm 16^{\circ}$ & $\sim 0.177$ \\
$\# 1 \mathrm{~b}$ & 10 & $7.5 \pm 0.4$ & $\sim 7.9$ & $\pm 9.5^{\circ}$ & $\sim 0.106$ \\
& 25 & $7.8 \pm 0.4$ & $\sim 7.0$ & $\pm 10.5^{\circ}$ & $\sim 0.117$ \\
\hline
\end{tabular}

fitted to:

$$
S=A \exp \frac{-\left(\lambda-\lambda_{0}\right)^{2}}{2 \sigma_{\lambda}^{2}}
$$

where $\sigma_{\lambda}$ is the Gaussian width. The root mean square (RMS) velocity along the laser propagation axis $x, \sqrt{\left\langle v_{x}^{2}\right\rangle}$, can be calculated as $\frac{\sigma_{\lambda}}{\lambda_{0}}=\sqrt{\frac{\left\langle v_{x}^{2}\right\rangle}{c^{2}}}$ while the mean transverse kinetic energy is $E_{x}=\frac{1}{2} m_{\mathrm{Ps}}\left\langle v_{x}^{2}\right\rangle$, where $c$ is the speed of light and $m_{\mathrm{Ps}}$ the mass of Ps [53].
The RMS Ps velocities (mean Ps energies) obtained from the Doppler profiles measured in \#1a are $(6.3 \pm 0.5) \times$ $10^{4} \mathrm{~m} \mathrm{~s}^{-1}(22 \pm 4 \mathrm{meV})$ for a laser delay of $10 \mathrm{~ns}$ and (4.4 $\pm 0.4) \times 10^{4} \mathrm{~m} \mathrm{~s}^{-1}(11 \pm 2 \mathrm{meV})$ for a delay of $25 \mathrm{~ns}$. The kinetic energy measured at $25 \mathrm{~ns}$ corresponds to the complete thermalization of Ps in the direction of the laser in the target kept at RT. The different velocities at different laser delays can be explained with the fact that the UV laser pulse, thanks to its brief duration and its limited spatial dimension, excites only 
the fraction of the emitted Ps in front of the target at the time when the laser is shot [36]. Thus, the most delayed laser pulse addresses Ps that resided longer in the nanochannels losing more energy by collision with the walls. The RMS Ps velocities (mean Ps energies) measured in \#1b are higher than the ones in \#1a: $(7.9 \pm 0.5) \times 10^{4} \mathrm{~m} \mathrm{~s}^{-1}(35 \pm 5 \mathrm{meV})$ for a delay of $10 \mathrm{~ns}$ and $(7.0 \pm 0.7) \times 10^{4} \mathrm{~m} \mathrm{~s}^{-1}(28 \pm 6 \mathrm{meV})$ for $25 \mathrm{~ns}$. This increase of the velocities (energies) could arise from the previously mentioned Ps reflection from the nanochannel bottom end that could induce a slightly shorter permanence time of Ps inside target \#1b compared to in \#1a with a consequent less efficient cooling.

The fitted $S$ value of the $1^{3} \mathrm{~S} \rightarrow 2^{3} \mathrm{P}$ peak at resonance (figure 7) is $10.4 \pm 0.8$ and $13.9 \pm 1.0$ for converter \#1a at 10 and $25 \mathrm{~ns}$, respectively. In \#1b, it is $7.5 \pm 0.4$ and $7.8 \pm 0.4$ at 10 and $25 \mathrm{~ns}$, respectively (see table 1). These values can be roughly approximated as the following product:

$$
S[\%]=100 \% * d * s * \eta * \varepsilon,
$$

where $d$ is the excitation efficiency imposed by the bandwidth coverage of the UV laser, $s$ the saturation efficiency of the $1^{3} \mathrm{~S}$ $\rightarrow 2^{3} \mathrm{P} \rightarrow$ ionization process, $\eta$ is the laser coverage of the solid angle of the Ps cloud and $\varepsilon$ is the fraction of Ps within the measured velocity range.

The UV pulse, due to its bandwidth of $\sim 50 \mathrm{GHz}$, selectively excites only the emitted Ps with a velocity component parallel to the laser propagation axis, $v_{x, \mathrm{BW}}$, with $\left|v_{x, \mathrm{BW}}\right|<$ $1.3 \times 10^{4} \mathrm{~m} \mathrm{~s}^{-1}$. Thus, only Ps expanding within a spherical wedge, whose angle is determined by the Ps average velocity, is addressable (see also references $[8,35]$ ). The wedge angles calculated as $\theta= \pm \arctan \frac{\left|v_{x, \mathrm{BW}}\right|}{\sqrt{\left\langle v_{x}^{2}\right\rangle}}$ for the different experimental conditions are reported in table 1 . From previous measurements, Ps emission is expected to be essentially isotropic in velocity [34, 36, 38]. A further evidence of the isotropy of the Ps velocity comes from the $S$ scan of figure 6 where the excitation signal in \#1a disappears after around $70 \mathrm{~ns}$ from the positron implantation. As Ps has to travel around 3-4 $\mathrm{mm}$ to cross the UV laser spot (see UV FWTM given in section 2.3), the velocity needed to cover this space within $70 \mathrm{~ns}$ is $\sim 4.2-5.7 \times 10^{4} \mathrm{~m} \mathrm{~s}^{-1}$, in good agreement with the measured transverse velocity in this target. In the light of the Ps velocity isotropy, the fractions of Ps expanding within the spherical wedges with the reported angles can be approximated as $d=\frac{2 \theta}{\pi}$. The values of $d$ for the two converters for both delay times are reported in table 1 .

Taking into account the energy of the UV and VIS laser pulses used in the present scan and according to the observation of the saturation of both UV and VIS energies shown in figures 3(b) and (c), the term $s$ gets closer to 1. Given the dimensions of the laser and the $\mathrm{e}^{+}$beam and the alignment of the laser grazing the target, the laser coverage of the solid angle of the Ps cloud, $\eta$, is expected to be not far from unity. If one assumes $s$ and $\eta=1$, equation (3) can be simplified to $S[\%]=100 \% * d * \varepsilon$ and a lower bound for the fraction of Ps within each measured velocity range, $\varepsilon$, can be roughly estimated from the fitted $s$ values and the geometrical evaluations of $d$ (table 1 ). We find that the fraction of Ps with average velocity of $\sim 4.4 \times 10^{4} \mathrm{~m} \mathrm{~s}^{-1}$ (measured in \#1a at $25 \mathrm{~ns}$ ) is, at least, $\varepsilon \sim 0.8$ of the entire emitted Ps. As the emitted Ps from this target is around $35 \%$ of the implanted $\mathrm{e}^{+}$at $3.3 \mathrm{keV}$ (from fit of figure 4), $28 \%(=35 \% \times 0.8)$ of $\mathrm{e}^{+}$implanted in the converter \#1a kept at RT is emitted as completely thermalized Ps. Similarly, the fraction of Ps with average velocity of $\sim 6.3 \times 10^{4} \mathrm{~m} \mathrm{~s}^{-1}$ (measured in \#1a at $10 \mathrm{~ns}$ ) is, at least, $\varepsilon \sim 0.8$ of the entire emitted Ps. The fact that $\varepsilon \sim 0.8$ in the two cases means that, in the time elapsed between $10 \mathrm{~ns}$ and $25 \mathrm{~ns}$, there is a balance between the fast Ps fraction escaping from the laser spot and the fraction, with lower velocity, that enters the laser spot after delayed emission from the target. For sample \#1b, we find that at least $\varepsilon \sim 0.7$ and $\sim 0.65$ are emitted with an average velocity of $\sim 7.9 \times 10^{4} \mathrm{~m} \mathrm{~s}^{-1}$ and $\sim 7.0 \times$ $10^{4} \mathrm{~m} \mathrm{~s}^{-1}$, respectively.

\section{Conclusion}

The data presented here demonstrate the possibility to overcome the cooling limitations of Ps in nanochannels by morphological tuning of the pores. Such limitations are the quantum confinement, that hinders the cooling of Ps below a certain threshold in small pores $[13,30]$ and the Ps interaction rate with the inner surfaces of the pores that, in large open volumes, becomes too low to guarantee an efficient cooling $[27,29]$. These limitations were overcome by exploiting the possibilities of adjusting both the diameter and the length of nanochannels offered by electrochemical etching in silicon.

An efficient source of Ps with kinetic energy of $11 \pm 2 \mathrm{meV}$, corresponding to the complete thermalization of Ps in the converter kept at RT, has been realized by producing nanochannels with a diameter of 7-10 nm and a length of $\sim 3.89 \mu \mathrm{m}$. The amount of thermal Ps has been roughly estimated to be $\sim 28 \%$ of the $\mathrm{e}^{+}$implanted at $E=3.3 \mathrm{keV}(\langle z\rangle \sim 140 \mathrm{~nm})$. The reduction of the nanochannels length has resulted in an incomplete thermalization of the Ps but in a higher Ps conversion efficiency due to contribution coming from Ps reflected from the bottom of the channels.

The production of a large amount of cold Ps is required in a broad range of experiments. Among them, the anti-hydrogen production via charge exchange reaction [9-11] will directly benefit from the efficient source of Ps at $\sim 11 \mathrm{meV}$ demonstrated here thanks to the higher cross section reaction guaranteed by the employment of slower Ps [56]. Moreover, slower Ps allows exciting Ps to higher Rydberg levels in strong magnetic fields avoiding the self-ionization rate due to the motionalStark effect $[38,57,58]$. The use of the present $\mathrm{e}^{+} /$Ps converter will be of direct benefit also for the production of a beam of $2^{3} \mathrm{~S}$ metastable Ps suitable for inertial sensing experiments on this matter/antimatter leptonic system [8]. Finally, the converter characterized here could be of help in highprecision QED experiments [59]. Tests of the Ps emission kinetic energy from the target kept at cryogenic temperature are planned to verify if slower Ps can be produced by reducing the converter temperature and tuning the positron implantation energy. 


\section{Acknowledgments}

We acknowledge Dr. N Bazzanella for his kind availability for SEM measurements. We also thank Prof. P Bettotti for the useful discussions about nanochannelled silicon. This work was supported by Istituto Nazionale di Fisica Nucleare; the CERN Fellowship programme and the CERN Doctoral student programme; the Swiss National Science Foundation Ambizione Grant (No. 154833); Marie Sklodowska-Curie Innovative Training Network Fellowship of the European Commission's Horizon 2020 Programme (No. 721559 AVA); European Research Council under the European Unions Seventh Framework Program FP7/2007-2013 (Grants Nos. 291242 and 277762); European Union's Horizon 2020 research and innovation programme under the Marie Sklodowska-Curie Grant Agreement ANGRAM No. 748826; Research, and Economy; Research Council of Norway under Grant Agreement No. 303337; Bergen Research Foundation; John Templeton Foundation; Ministry of Education and Science of the Russian Federation and Russian Academy of Sciences and the European Social Fund within the framework of realizing Research infrastructure for experiments at CERN, LM2015058; the European Union's Horizon 2020 research and innovation programme under the Marie Sklodowska-Curie Grant Agreement No. 754496-FELLINI.

\section{Data availability statement}

The data that support the findings of this study are available upon reasonable request from the authors.

\section{ORCID iDs}

S Mariazzi (D) https://orcid.org/0000-0001-9985-8792

B Rienäcker (D) https://orcid.org/0000-0002-3836-7030

A Camper (iD) https://orcid.org/0000-0002-8664-3693

G Bonomi (D) https://orcid.org/0000-0003-1618-9648

G Consolati (D) https://orcid.org/0000-0003-3614-245X

L Povolo (D) https://orcid.org/0000-0003-1451-1947

\section{References}

[1] Deutsch M 1951 Phys. Rev. 82455

[2] Rich A 1981 Rev. Mod. Phys. 53127

[3] Cassidy D B 2018 Eur. Phys. J. D 7253

[4] Mills A P and Leventhal M 2002 Nucl. Instrum. Methods Phys. Res. B 192102

[5] Oberthaler M K 2002 Nucl. Instrum. Methods Phys. Res. B 192 129

[6] Crivelli P, Cooke D A and Friedreich S 2014 Int. J. Mod. Phys. Conf. Ser. 301460257

[7] Cassidy D B and Hogan S D 2014 Int. J. Mod. Phys. Conf. Ser. 301460259

[8] Mariazzi S, Caravita R, Doser M, Nebbia G and Brusa R S 2020 Eur. Phys. J. D 7479

[9] Humberston J W, Charlton M, Jacobson F M and Deutch B I 1987 J. Phys. B: At. Mol. Phys. 20 L25
[10] Doser M et al (AEgIS Collaboration) 2012 Class. Quantum Grav. 29184009

[11] Pérez P et al (GBAR Collaboration) 2015 Hyperfine Interact. 23321

[12] Mariazzi S, Toniutti L, Patel N and Brusa R S 2008 Appl. Surf. Sci. 255191

[13] Cassidy D B, Crivelli P, Hisakado T H, Liszkay L, Meligne V E, Perez P, Tom H W K and Mills A P Jr 2010 Phys. Rev. A 81 012715

[14] Liszkay L et al 2012 New J. Phys. 14065009

[15] Mariazzi S, Bettotti P, Larcheri S, Toniutti L and Brusa R S 2010 Phys. Rev. B 81235418

[16] Mariazzi S, Bettotti P and Brusa R S 2010 Phys. Rev. Lett. 104 243401

[17] Paulin R and Ambrosino G 1968 J. Phys. France 29263

[18] Sferlazzo P, Berko S and Canter K F 1985 Phys. Rev. B 32 6067

[19] Sferlazzo P, Berko S and Canter K F 1987 Phys. Rev. B 355315

[20] Nagashima Y, Morinaka Y, Kurihara T, Nagai Y, Hyodo T, Shidara T and Nakahara K 1998 Phys. Rev. B 5812676

[21] Van Petegem S, Dauwe C, Van Hoecke T, De Baerdemaeker J and Segers D 2004 Phys. Rev. B 70115410

[22] Chang T, Xu M and Zeng X 1987 Phys. Lett. A 126189

[23] Vallery R S, Zitzewitz P W and Gidley D W 2003 Phys. Rev. Lett. 90203402

[24] Ito K et al 2005 J. Appl. Phys. 98094307

[25] Tanaka H K M, Kurihara T and Mills A P Jr 2006 J. Phys.: Condens. Matter 188581

[26] He C, Ohdaira T, Oshima N, Muramatsu M, Kinomura A, Suzuki R, Oka T and Kobayashi Y 2007 Phys. Rev. B 75 195404

[27] Nagashima Y et al 1995 Phys. Rev. A 52258

[28] Tuyen L A et al 2020 Radiat. Phys. Chem. 172108867

[29] Cassidy D B, Hisakado T H, Meligne V E, Tom H W K and Mills A P Jr 2010 Phys. Rev. A 82052511

[30] Mariazzi S, Salemi A and Brusa R S 2008 Phys. Rev. B 78 085428

[31] Bisi O, Ossicini S and Pavesi L 2000 Surf. Sci. Rep. 381

[32] Chuang S F, Collins S D and Smith R L 1989 Appl. Phys. Lett. 55675

[33] Guatieri F, Mariazzi S and Brusa R S 2018 Eur. Phys. J. D 72 198

[34] Aghion S et al (AEgIS Collaboration) 2016 Phys. Rev. A 94 012507

[35] Aghion S et al (AEgIS Collaboration) 2018 Phys. Rev. A 98 013402

[36] Amsler C et al (AEgIS Collaboration) 2019 Phys. Rev. A 99 033405

[37] Antonello M et al (AEgIS Collaboration) 2019 Phys. Rev. A 100063414

[38] Antonello M et al (AEgIS Collaboration) 2020 Phys. Rev. A 102013101

[39] Cassidy D B, Deng S H M, Tanaka H K M and Mills A P 2006 Appl. Phys. Lett. 88194105

[40] Cassidy D B and Mills A P 2007 Nucl. Instrum. Methods Phys. Res. A 5801338

[41] Brusa R S, Macchi C, Mariazzi S and Karwasz G P 2005 Acta Phys. Pol. A 107702

[42] Zhang X G 2004 Etching of silicon Electrochemistry of Silicon and its Oxide (Berlin: Springer)

[43] Zecca A, Bettonte M, Paridaens J, Karwasz G P and Brusa R S 1998 Meas. Sci. Technol. 9409

[44] Macchi C, Mariazzi S, Karwasz G P, Brusa R S, Folegati P, Frabboni S and Ottaviani G 2006 Phys. Rev. B 74174120

[45] Soininen E, Schwab A and Lynn K G 1991 Phys. Rev. B 43 10051

[46] Cooper B S, Alonso A M, Deller A, Liszkay L and Cassidy D B 2016 Phys. Rev. B 93125305 
[47] Mills A P and Gullikson E M 1986 Appl. Phys. Lett. 49 1121

[48] Danielson J R, Dubin D H E, Greaves R G and Surko C M 2005 Rev. Mod. Phys. 87247

[49] Penasa L, Di Noto L, Bettonte M, Mariazzi S, Nebbia G and Brusa R S 2014 J. Phys.: Conf. Ser. 505 012031

[50] Aghion S et al (AEgIS Collaboration) 2015 Nucl. Instrum. Methods Phys. Res. B 36286

[51] Yzombard P 2016 PhD Thesis Université Paris-Saclay https:// tel.archives-ouvertes.fr/tel-01446588/document

[52] Andersen S L, Cassidy D B, Chevallier J, Cooper B S, Deller A, Wall T E and Uggerhøj U I 2015 J. Phys. B: At. Mol. Opt. Phys. 48204003
[53] Gurung L, Alonso A M, Babij T J, Cooper B S, Shluger A L and Cassidy D B 2019 J. Phys. B: At. Mol. Opt. Phys. 52105004 [54] Petkov M P, Wang C L, Weber M H, Lynn K G and Rodbell K P 2003 J. Phys. Chem. B 1072725

[55] Guatieri F 2018 PhD Thesis University of Trento http://eprintsphd.biblio.unitn.it/2954/

[56] Krasnicky D, Caravita R, Canali C and Testera G 2016 Phys. Rev. A 94022714

[57] Castelli F, Boscolo I, Cialdi S, Giammarchi M G and Comparat D 2008 Phys. Rev. A 78052512

[58] Amsler C et al (AEgIS Collaboration) 2021 Commun. Phys. 4 19

[59] Gurung L, Babil T J, Hogan S and Cassidy D B 2020 Phys. Rev. Lett. 125073002 